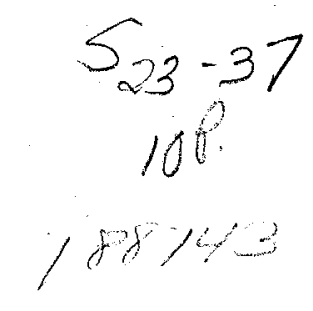

\title{
A Universal Six-Joint Robot Controller
}

\author{
D.G. Bihn \\ Hewlett-Packard \\ Cupertino, CA 95104 \\ T.C. Hsia \\ University of California, Davis \\ Davis, CA 95616
}

\section{Abstract}

A general purpose six-axis robotic manipulator controller was designed and implemented to serve as a research tool for the investigation of the practical and theoretical aspects of various control strategtes in robotics. A 80236-based Intel System 310 running the Xenix operating servo sof tware as well as the higher level sof tware (e.g. Kinematics and path planning). A Multibus compatible interface board was designed and constructed to handle 1/0 stgnals from the robot manipulator's joint motors.

From the design point of view, the universal controller is capable of driving robot manipulators equipped with D.C. joint motors and positton optical encoders. To test its functionality, the controller is connected to the joint motor D.C. power amplifier of a PUMA 560 arm bypassing completely the manufacturer-supplied Unimation controller. A controller algorithm consisting of local PO control laws was written and installed into the $x$ enix operating system. Addticical sof tware drivers were implemented to allow application programs access to the interface board. All software was written in the $C$ language.

\section{Introduction}

Robots are beconing increasingly prevalent in the industrial workplace, as well as creating an industry of their own. This new industry is both driving and being dofven by new technologles. New matertals, improved their own. This new ind faster controller electronics ape running into the limitations of traditional control mechanical Thus, theoretical work to overcome these fimitations is urgently needed. Much of the theoretical work is being carried out in academic research institutions. However, there is of ten a significant gap between the results of theoretical studies based on simulations and the verification based on actual implementation. industry is of ten reticent to try untested theore:tcal results, preferring the time-tested, sub-optimal control techniques of the past, possibly sacrificing substantial performance improvements. A credible testing ground for new control techniques is needed to bridgé the gap between theory and application.

The Robotics Research Laboratory at the University of California, Davis, has a Unimation PUMA 560 rooot arm reoresentative of a large and popular class of modern industrial manipulators. The PUMA arm is controlled representate sophisticated robot ianguage, VAL-Il. The user only has access to the arm through high level 'move-type' comnands. He therefore has iftele control of the actual arm trajectory and no control over the low level motor servo loops. In typical industrial applications, the inability to alter low level functions of the controller does not represent a functional limitation. To the contrary, it actually affords both the arm and the operator a fair degree of protection and safety. The acadenic researcher. however. is prevented fran using the am to test and demonstrate new control strategies and is forced to rely on computer simulation.

\section{Objective}

The objective of this project was to design and implement a cimputer based robotic controller which allows the researcher to write programs and implement algorithms which coitrol the robot arm from the lowest level of the closed-loop servo system to the higher levels of kinematics, dynamics, path planning and robct language [11]. The use of a familiar sof tware environment was chosen with the intent of making the user interface as cledi and simple as possible.

The scope of this project is limited to the design and implementation of a controller consisting of (1) the Joint Interface Board electronics, and (2) the operating system interface to this hardware. A simple low level 6-joint P.I.D. (Proportional Integral Derivative) controller is implemented and presented to serve as both a functional test of the system and as an application example. The topics of joint kinematics and other high level application sof tware are beyond the scope of this project as is the advanced control law design. 


\section{The Controller System}

The contrciler presented here is designed around an Inte1 310, 80286-based, microcomputer [2] running the UNIX-ifke operating system, XENIX [3]. A signal interface board was designed and constructed to provide the interface between the microcomputer and the jol nt motors of the arm. The Unimation controller, supplied as part of the PUMA 560, was modiffed to serve two low level functions: as a convenient access point for the jolnt feedback signals from the arm and as a multi-channel power amplifier drive the jolnt motors. All other electronics in the Unimation multi-channel power amplifier to drive the joint motors. All other electronics in the Unimation controller are by-passed; closed-loop control is done in the Intel-based controller described here. The controlier system is depicted by the block diagran shown in figure 1.

A single $80286 \mathrm{CPU}$ runnting at $6 \mathrm{MHz}$ is used to execute both high level (e.g. kinematics) and low level (e.g. joint servo loops) control sof tware. At a typical sampling rate of $100 \mathrm{~Hz}$, about $30 x$ of the CPU time ts required to execute the six P.I.D. controllers implemented in the design example. The remaining CPU time is avaliable for application programs and the operating system. The interface board itself is useful in systems with sampling rates over $2 \mathrm{KHz}$. However, to utilize this speed, additional CPU power is required.

\section{System Design Requil rements}

Two basic elements constitute the controller system designed and implemented in this project: a digital computer and spectal purpose interface hardware. The digftal computer performs all the control functions. from the joint motor servo control law to the higher levels of coordinated joint motion. The faterface hardware function is to provide the basic link between the computer and the physical signals requíaj to cintrol the robot arm.

\subsection{The D.C. Serva Motor Posttion Measurement}

The control of the robot arm is equivalant to the control of the joint motors. In this controller, D.C. servo motors are assimed to be equipped with potentiameter and/or incremental encoder position feedback devices. It is also assumed that the D.C. motor can be driven by an analog (voltage) signal buffered by an appropriate external power amplifier (servo motor amplifier). The Untmation PUMA $560 \mathrm{arm}$ has six geared D.C. Servo motors with both encoder and potentiomenter position feedback elements and it is considered to be prototypical of the class of manipulators considered in this project.

Each motor, in general, does not directly drive a manipulator joint, but is typically connected through a gear train requiring a multiple number of motor revolutions to ortve the joint through its operating iange as shown in Figure 2. In the configuration assumed in this project, feedback elements are directly attached to shewn motor, not the actual joint member. Joint position is inferred from the motor position. This requires that absolute motor position must be measured over multiple revolutions. In the PUMA arm, both a geared (i.e. muititurn) potentiameter and an incremental shaft encoder are connected to the motor shaft to co'lectively supply this data. The incremental encoder is used to accurately measure both the relative motor position over an arbitrary number of rotations and the absolute motor positton modulo one rotation. The geared potentioneter is used to measure the approximate absolute motor angle over the several revolutions needed to drive the joint through its range. Once the absolute motor angle has been determined, only the relative data supplied by the encoder is needed.

The incrementai encoder, which is directly attached to the motor, generates two types of data: (1) high resolution quadrature signals which are decoded into relative (incremental) angular displacement information and (2) an index pulse which is produced once per revolution and can be used to accurately define the absolute angular position of the motor modula $360^{\circ}$ (Ffgure 2).

The geared potentiometer supplies indirect, low resolution absolute joint position data. The gear ratio of the potentioneter is designed so that when the joint is driven between its mechanical limits, the pot wiper rotates within its mechanical limits (less tilan $360^{\circ}$ ). Logically, this pot could have been attached directly to the robot joint. For manufacturability considerations, the pot has been included in the motor assembly.

Once the absolute motor position has been determined, it is continuously updated (increnented or decremented) by the data from the incremental shaft encoders. As long ds the electronics are not interrupted (e.g. power-down) the data from both the geared pot and the encoder's index pulse are not used. The difficulty. is the initial determination of the absolute motor position is rather involved and will now be discussed.

When the absolute motor position is unknown, the potentfometer wiper voltage can be measured and the absolute motor position estimated. Once estimated, further position measurement can be made by monitoring the relative position data from the incremental encoders. While the incremental data is very accurate, the absolute position can only be as good as the initial estimate. The standard technique to obtain an accurate measurement of the intial absolute position is as follows. First, the motor is driven until the encoder's index pulse is found. At this point the absolute position is known to be an exact multiple of $360^{\circ}$. Next, the potentioneter voltage is measured to give the approximate absolute position. Combining the approximate absolute position with the certain knowledge that the position is an exact multiple of $360^{\circ}$, the exact absolute can then be derived.

The above explanation serves to demonstrate the basic idea and what sort of precision is required. For analysis, the actual parameters of the PUMA 560 joint motors are used to determine the system specifications and Joint Interface Board requi rements. 


\subsection{A Iypical D.C. Servo Motor}

The PuMA 560 servo motors are integral packages which contain four basic components: (1) a 0.C. motor: (2) an electric brake; (3) an optical incremental encoder; and (4) a geared-down potentiometer. The currents activating the motor and the electric brake are the inputs while the encoder and the potentiometer signals are the outputs. The basic functions needed to operate the motor system are described below.

\subsubsection{Reading the Incremental Encoder}

The Incremental encoder has three output signals: channels $A, B$, and the index pulse. Channels $A$ and $B$ are used to determine both the amount and direction of rotation in discrete steps. The index pulse produces a single short pulse each motor revolutton whtch can be used by the system to determine the absolute angle of the motor and, with the addition of the potentfometer data, can be used to determine absolute positfon (described above).

The output sicates of channels $A$ and $B$ are used to detect relative motion (rotation) of the motor shaft and in turn, the joint itself. How this is done is well-known and is not described here.

\subsubsection{Counting the State Changes}

The incremental encoders on the PUMA 560's motors produces 1000 state transitions per revolution, except for the shoulder joint (\#2) which produces 800 transitions. The motor (with the encoder directly attached) rotates from 40 to 60 times during full joint travel (Jepending on the joint), corresponding to 40,000 to 60,000 rotates from 40 to 60 tiete joint motion. It is convenient if the hardware keeps count of the total joint range. This way the total joint motion may be read dfrectly from the hardware counters. 16-bit counters have a maximum count of 65,532 and are sufficient to keep track of the joint motors of the PUMA arm. However, it is not essential for the hardware to count the entire joint range. In a sampled data system, the sof tware can keep track of total joint motion, using the hardware only to count the relative motion which has occurred between samples. If the hardware count is used in this manner. the absolute motion is limited only by sof tware and the incremental motion between samples is limited to motion of $\pm 32 \mathrm{~K}$ fulses.

\subsubsection{Reading the Potentiometers}

The potentiometers incorporated into the PUMA 560 joint motors are connected between +5 volts and ground. Rotating the pot through $360^{\circ}$ produces a proportional voltage output from 0 to 5 volts (e.g., $90^{\circ}$ produces 1.25 volts, $180^{\circ}$ produces 2.5 volts, etc.). These pots ha': been geared so that they rotate somewhat less than $360^{\circ}$ volts, a complete joint movement; depending on the joint, full joint travel may produce as little as $200^{\circ}$ of potentiometer motion. This restricted travel corresponds to a change in pot voltage of about 2.78 volts. If the joint produces 60 index pulses (i.e. 60 motor rotations) per full joint motion, the pot voltage must be measured to an absolute accuracy of $1 / 60$ th of 2.78 volts $(0.046$ volts) in order to determine the motor shaft angle to within one revolution.

An Analog to Digital Converter $(A D C)$ is used to measure the pot voltages. It must be able to measure a voltage which spans a 0 to 5 volt range, and must have a resolution and accuracy of better than 0.046 volts over this range. This corresponds to a full-scale resolution of $0.92 \%$. A seven-bit ADC has a resolution of $0.78 \%$ and is sufficient for this voltage measurement.

Since the potentimeters are not part of the dynamic control scheme presented here, there is no constratint on the conversion speed. For the PUMA arm, both speed and resolution requirements of the ADC are easy to meet. However, to make the system more flexible, other possible applications should be considered. It is of ten the case where a symmetrical voltage signal (say -5 to +5 volts) needs to be measured and fast conversion time can make dynamic control systems with anal og feedback elements possible. Furthermore, since fast (30 microsecond) 12 -bit ADCs with input range of \pm 5 volts are conventently available and at reasonable cost. this higher performance device was chosen.

\subsubsection{Oriving the Motor}

The drive current and voltage needed by a D.C. motor depends on the stze and type of motor used; no solution is appropriate for all motors. It is therefore considereu impractical to include the porer amplifier as part of the design. The important requirement is how to drive these power ampliffers.

In general, two standard techniques for supplying the current needed for driving $0 . C$. servo motors are commonly used: Iinear amplifiers and pulse width modulated (PWM) amplifiers. Each have advantages but the important fact tc consider is that they both are controlled by a simple analog voltage.

In the particular case of the PUMA 560 arm, the Unimation PUMA controller's power amplifiers can be conventently used because they have been designed explicitly to drive the PUMA 560 joint motors. Using this controller also makes the external connection to the arm joint motors simple and straightforward. Additiona!ly, the Unimation amplifier has several useful safeguards which automatically shut the amplifier off to prevent damage to the arm.

Power amplifiers are controlled by analog voltages, and to generate these voltage outputs from a digital controller a Digital to Analog Coriverter. (DAC) must be used. Three basic specifications must be considered: (1) voltage swing: (2) resolution (number of bits); and (3) the accuracy. Commercially available power amplifiers typically require a vol tage input of -10 volts to +10 volts. This also corresponds to typical DAC device output characteristics, and the input specifications of the Unimation controller's power amplifier. 
Selection of resolution and accuracy is more difficult. 8-bit corresponds to a resolution of une part in 256 $(0.39 \%)$, 10-bit corresponds to one part in $1024(0.098 \%)$, and 12-bit corresponds to one part in 4096 (0.024\%). A 10-bit unt was chosen and considered to be a reasonable compromise between price and performance.

\subsubsection{Releasing the Brake}

The brake is used to lock each joint in position when the servo motor is turned off and is necessary to keep the arm from collapsing. The brakc is much like a $0 . C$. rel ay. When 0.C. current passes through the coll (an electromagnet). the brake plate is retracted from the friction plate allowing the motor to rotate. When no current is flowing in the brake coil, the brake plate is forced into contact with the friction plate by compression springs and the motor cannot rotate. On the Unimate controller, the brake release current is supplied whenever the 'arm power' is on. This is a fall-safe system. When the servo power (to the motors) 1s turned on, the brakes are released. When the arm power is switched of $f$, the brakes are automatically applifed, holding the joint in place.

\subsubsection{0ther $1 / 0$ Requi rements}

The Joint Interface Board must not only accommodate the joint motor signels but must also provide the host computer with additional functions to allow all subsystems to be integrated into a complete controller. included in the design are (1) a digftal timer and associated interrupt circultry, and (2) 24 bits of general purpose $1 / 0$ lines.

\subsection{Host Computer Reguirements}

The selection of suttable host computer is very important. The machine must not only be capable of meeting basic execution speed and $1 / 0$ requirements, but should also be able to support the sof tware tools needed to implement a controlier. In this section both the host computer hardware and sof tware are discussed.

\subsubsection{The Computer: Intel System 310}

The Intel System 310 microcomputer was used because it satisfies the above criteria. It is based on the intel 80286 16-bit microprocessor [4]: the systen also comes equipped with an 80287 floating point nath coprocessor [5]. It is a Mul tibus based system [6], a bus standard which is particularly popular in the area

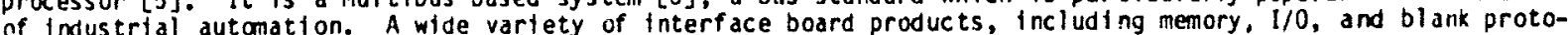
type boards, are avaflable from Intel and third party vendors. A standard Mul tibus board is comparattvely large which allows complex circuits to fit onto a single board, allowing the use of a single bus interface circult. All the hardware for the Joint interf ace Board was able to fit on a single board.

\subsubsection{The Operating System Chotce: XENIX 286}

The Intel 310 can run several operating systems: the ubiquitous IBM PC's MS-DOS, the UNIX-1ike XENIX system 0.S.. and the real-time, multi-tasking systems RMX-86 and RMX-286.

XENIX was chosen to be the operating system of this project's fmplementation. A substantial learning effort is required to become profictent with an unf amiliar operating system and new sof tware tools. XENix minimizes this obstacle; many researchers are familitar with UNIX and need little time to master XENIX. Those unacquainted with UNIX can be motivated to learn XENIX since this knowledge is useful on many other systems. This is a very tmportant consideration on short term projects where learning a new operating system may require more time than the experiment itself.

The XENIX operatinn system is Microsoft's licensed version of UNIX III with some of the Berkeley Sof tware Distribution (BSD) enhancements (e.g. ' $v i$ ' and the $C$-shell), and several of their own enhancements. It is a multi-user system. UNIX is a very pawerful enviroment for developing sof tware and is widely used in the acadenic and research comentites. The disadvantage is that it was not designed for real-time applications. Details of the techniques used to construct a real-time controller for our purpose are given later.

\section{The Design}

This section details the design and implemenzation of the above specifications. The discussion is divides into three sections: (1) the hardware design of the Joint Interface Board (JIB); (2) the connection between the J.i.B. and the Unimate PUMA 560 controlier; and (3) the suftware interface between the kitix operating system ano the JIB.

\subsection{Joint Interface Board Design}

The block diagram which outlines the J.I.o. hardware is shown in Figure 3 . As seen fram the computer side of the bus interface, the JIB is a small collection of $I / 0$ devices: six 16-bit encoder counters; ar. encoder reset circuit; two PIO (parallel input/output) devices; timer and the interrupts reset logic. One of the PIOs is used exclusively to interface to the ADC and DAC subsystem, and the other PIO is used for off-board digital expanstion. 


\subsubsection{The And og-01yital ذubsystem}

Communicatien and ontrol sianils for $A l l$ seven DACs, the AOC and the analog multiplexer ar........ to

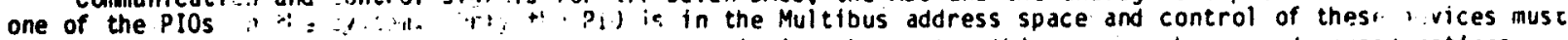
be made through tili: $i$ : The PIO, an 8255 , can operate at 5 . full 80286 bus speed while the ADCs and DACs are about twice as slow (450 ns vs $180 \mathrm{~ns}$ ). Rather than $-:$ ow the bus town on this board and degrade performance of the other onboard devices (e.g. the encoders), the ADC and 'Acs a given their own private slow bus.

\subsubsection{Analog Output: The DACs}

Six analog voltage outputs are necessary to drive the basic joint servo motors. An additional analog vol tage output was included to permit future expansion, possibly the control of a mure sophisticated gripper. To produce these outputs, seven independent DACs (digital to analog converters) were used. The 1 ndependent DAC approach offers the advantaye of a very straightforward interface. improved accuracy and simpler ctrcuit design.

As described in the Analysis section above, the analog outputs must be capable of delivering a voltage from -10 volts to +10 volts at a resolution of 10 bits (1 part in 1024) to properly drive the inputs of the servo motor power amplifier.

\subsubsection{Anal og Input: The ADCs}

As described in the analysis section, each of the PUMA 560 joint motors has a potentianeter which produces an output from 0 to 5 volts and, to be useful in absolute position determination, these signals must be resolved to an 8-bit accuracy. Fast, high resolution analog to digital converters can be obtained at reasonable prices which exceed the basic specification but give the Jot nt interface Board more power. Analog Devices' AD574 [7] is a popular example. It has a 12-bit resolution, a converston speed of less than 30 micro-seconds, selectable input ranges of 0 to $+10,0$ to $+20,-5$ to +5 , and -10 to +10 volts, and a cost of less than 335 . At this speed of conversion, one device is fast enough to convert all six jotnts pot data in less than 0.2 milliseconds, a speed fast enough to allow the pots alone to be used as the primary feedback element in situations where it may be useful.

To use one $A D C$ to convert several analog input signals requires the use of an analog selector or multiplexer. A typical analog multiplexer, the IF 1308 has eight voltage inputs which are selected to one output. This output can then be converted by the ADC, one at a time. Like the DAC outputs, ADC outputs must also be latched. However, since the ADC output is digital, it may be easily stored inside the computer using software without using any spectal hardware.

\subsubsection{Timer Subsystem}

Generating a constant sampling interval requires an external clock source to interrupt the CPU and cause the control sof tware to execute. The Multibus provides a $10 \mathrm{MHz}$ clock requiring an onboard frequency divider logic. To allow convenient changing of the sampling rate, a divide-by-ten prescaler followed by a microprocessor compatible programmable timer was selected. An Intel I8254 triple 16-bit timer i.C. [8] was used. it features extensive programability, high resolution (one part in 65K). In the divide-by-n mode it can be

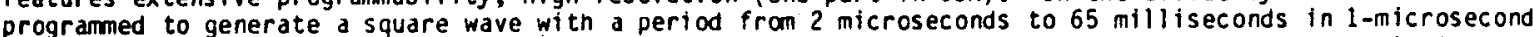
steps. This corresponds to a rate from $22 \mathrm{~Hz}$ to $500 \mathrm{kHz}$ (though rates above $200 \mathrm{~Hz}$ are not us able in the present system). Timer $\# 0$ is used as the interrupt clock, leaving timers $\# 1$ and $\# 2$ available for future applications.

\section{1 .5 Digital $1 / 0$}

To make the Joint Interface Board a more flexible and general purpose interface, an additional parallel input/output (PIO) I.C. was included in the design. All the 24 outputs from this device go directly of $f$-board via the connector $j 12$ and are not used by any of the onboard electronics.

\subsubsection{The Encoder Subsystem}

The JIB accepts six sets of incremental encoder signals. Each input set is used to control its own 16-bit counter, instructing it to count UP, count DOWN, do nothing, or RESET to zero. The encoder subsystem can be divided into three parts: (1) the basic up-down counter; (2) the decode logic; and (3) the reset logic.

\section{A. The Counters}

The 16-bit up-down counter is a straightforward cascading of four 4-bit up-down synchronous counter with three control inputs: clock enable (CE), up-down select (UD), and reset $(R)$. The system clock is running continuously at 1 MKz.

To directly implement a state decoder, six decoders would have to be constructed. This would probably require six 16-pin DIP packages. These would probably have to be either bipolar PROM (programmable read only memory) or some type of PLA (programmable logic array). If the PROM approach is used, a $16 \times 2=$ 32-bit PROM would be sufficient. The total number of bits required by all six units in this scheme is 196 bits.

This new 12-bit vector has 4096 possible states, each of which must be decoded to generate a 6-bit output vector. 0 , with the proper CE and UD signals for three counters. 
For six counters, a total of $4096 \times 6 \times 2=48 \mathrm{~K}$-bits is required. This is two orders of magnitude greater than the scheme where each counter has its own state decoder. The advantage of this bit wasteful approach is that all this decoaing can be done using just two 8 K-byte EPROMs packaged in 28-pin DIPs. These memory I.C.S are inexpensive and EPROM programmers are typically found in microprocessor developnent laboratories.

\section{B. The Encoder Reset System}

An index pulse signal is generated every incremental encoder (servo motor) rotation. This signal is used to supply quasi absolute position information about the motor so that the motor revolutions (e.g. $0^{\circ}$. $360^{\circ}, 720^{\circ}$, etc.) can be distinguished from one another. Typically these index signals are only used during initialization of the hardware and software after system power up. Once the system has been initialized, incremental information alone is sufficient to determine absolute position (provided no encoder state changes are missed).

The basic scheme of the reset/calibrate routine is to rotate each motor until the index pulse is found and then this position is defined to be the position zero. Conceivably, this could be done in software by continuously reading the index signal until it is detected. This would require the sof tware to sample the signal fast enough so that the pulse is not missed when the motor is moving at some speed.

To overcome this limitation, a hardware scheme was devised which allows calibration of the syste with the motor to be running at any speed within its operating limits. Each counter has a synchronous reset input. The index signal from the encoder could be connected to this input causing the particular counter to reset to zero whenever the index pulse occurs. However, since the motor typically rotates tens of times during the joint travel, some form of selectively gating the index signal on and of was required.

This circuit is asynchronously set or 'armed' via the ARM RESET signal. Once armed, the next occurrence of the index pulse generates a single reset pulse for the associated counter circuit. Once the reset pulse is issued, the circuit disarms itself so that further occurrences of the index pulse will not reset the counters. The sof tware can monitor these signals to check if the reset circuit is armed or not and can thereby detemine if the index pulse has occurred.

\subsubsection{The Multi-Bus Interface}

Up to this point, all the subsystems described here have been computer independent (except for the general requirement of a 16-bit bus). This allows easy conversion to many other 16-bit computers such as the 18M-AT. At this point the design becomes specific to the hardware of the host machine. The Intel 310 system is based on the Multibus. The Mul tibus supports direct addressability up to one megabyte through a 20 -bit address and 8and 16 -bit data transfers at a rate of five million transfers per second (10 MB/sec). The Joint Interface Board has been designed as a simple slave and never controis the Multibus. The JIB only decodes the address lires and acts upon the command signals from the bus master.

\subsection{The Unimation Interface}

The following sections describe how the Joint Interface Bodrd and the XENIX sof tware interface running on the Intel 310 were connected to the Unimate PUMA $560 \mathrm{drm}$. Position feedback signals from the arm servo motors are sent to the JIB, and the JIB sends analog voltage outputs to the power amplifier, which in turn drives the servo motor in each joint.

The Unimate PUMA controller consists of an LSI-11/73, six 6503-based joint controller boards, severai low level interface boards, and a six channel-high current power amplifier. The controller presented in this project makes use of only the power amplifiers and one of the feedback signal conditioning circuits. The LSi-1I and the six microprocessor joint controllers are completely bypassed.

To close the loop around the joint motors, the feedback signals from the PUMA 560 have to be connectad to the Intel system and the output command voltages must be returned from the Intel to the power amplifiers on the Unimate controller. It was considered desirabie to make the necessary modification to the unimate controiler in such a way that switching between the Intel controller and the internal Unimate controller systems is as sinpie and safe as possible.

Connecting the feedback signals from the Unimate Controller to the Joint Interface Board is accomplisned by inserting a proto-ijping card (fram nere on called the innimate interface Board) into one of the several a.jilable empty, unwired slots of the joint controller portion of the Unimate card cage [9]. This technique was selected for several reasons. All of the PUMA arm feedDack signals enter the controller through connectcr $j-30$ and are hard-wired directly to the ARM CABLE CARD in the card cage. Here some basic signal conditioning is performed, power is supplied to the joint pots and encoders, and the encoder outputs are then buffered to produce clean logic levels. Since these functions are required and would have to be duplicated if this siosystem was not used. it was convenient to use the external hardware' and obtain these signals after conditioning.

The only place these feedback signals dre found is in the backplane of the PUMA joint controller's cardcage. One of the available slots was chosen and all the necessary connections were made only by adding wires to the backplane, bringing all the feedback signals to the selected slot. This has the attractive feature of not having to break or cut any Unimate connections, leaving the controller intact. When the Unimation Interf ace card is removed fron its slot, the system is electrically and logically in its original condition. The card which is inserted into this slot also contains an inverting line driver to buffer the encoder signal to drive the wires connecting it to the Intel/JIB system. 


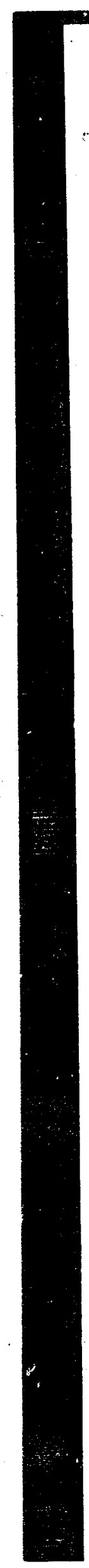

While the feecback signals can be sensed without breaking any connections in the unimate controller, this is not possible for the motor current command signals. In general. elther the Unimate or the Intel/JiB joint motor current command signals (the DAC outputs) can be used, since only one controller can be selectente trive any motor at any one time. Current command signals enter the power amplifier through connectors P73 ant Pla. These connectors are located on the top of the POWER AMP CONTROL card am are readily tetacher. Ihis is the only point where these signals can be 'intercepted' and the intel signals injected without permanently modifying the circuit (e.g. Cutting wires). A small interface panel with the appropriate connectors was faticatert to dllow the JiB and in imate motor current comans signals to he selectively sent to the linimate's power amplifier on a joint-by-joint basis (which s very useful during system debugging).

\subsection{The XEN1X to the Joint Coatrnller Bodrd Interfare.}

The Joint Interface Board is installed in the $1 / 0$ space of the Intel 310 (distinct from the memory space) and like dll other system hardware in XENIX, the usar can only access it through system device arivers. Drivers for all the JIB devices have been written ant installed into XEN!x (sep sof twere listing in Appendix $H$ [1]). Application programs access. these devices through symbolic names :e.j. "dac 1". "adc 4". "timer.l", etc.). The device ariver controls the details of the data format anef of physically addressing the nartware transparent to the application program $[10]$.

Properly written arivers protect the system from the application programs and make the user interface clean and simple. A motor controller can be implemented antirely th the application level, individually accessing the incremental encoders. UACs and the ADC through their respective device drivers. While this will work, much of the C.PI time is consimed in operating system overhead. Each $1 / 0$ request (e.y. read and write) takes substantidily longer to execuce than if the software is abie to directly address the hardware (not permitted in XEN(X). An alternative to lmplementing the controller at the application level is to piace it in the XENix karnel ds a single lolfical ripvice (e.j. rather than "dac l" and "encd l" devires, a single "pid l" can be considered as the basic l/1) init). Core written at the kernel level has direct access to the (/0 space and may red and write to the JIG wichout joing through the operating system. This reduction of overhead can reduce execiution time by abou: $50 \%$.

\subsection{Redl-Time 15sues}

xEidx is not a red-time operatiny system; it does not juarantee when a particular application progran will yet executed. It is of ten said that XENix (vis-a-vis UNix) does not guarantee when an interrupt is serviced. this only reters to the dpplication level. not the lowest level of interrupt handing. In the common applicition ot a interrupt is issued from the serial interface hardware (a UART) each time a rew character is received from the terminal. The interrupt handiling software then services the hardware, takin's the new chardctar and putilinit it into the terminal handler's buffer. This software only competes with other interrupt routines (e.j. other terminals) for (Plj time. Non-interrupt level operating system software which processes the charactiors in the termind handler's huffer must compete with the entire system (includinc jtrer anplication proyrams) for (epl] time and it is here where xeMlx cannot guarantee response time. This issd: is important in inslyninis a real-time controller.

A realy hasal real-time controller may be constructed in two fundamentally different ways. Both gethods remise that an axternal timer interrupt tho ceil de fixed intervals and that kernel level device diver be instaliat in the rfillx system to process this interrupt. In the first method, the interrupt handler of the irivar resuonits to the timer interrupt by only settini a flat in the driver's memory. when the 'device' is read by the tholication sof ware, the redo part of the ifriver tests this memory flay. if the flan is set, it returns har to the ablication vroirdr. If the lldij has not neen set (i.e. the timer has not yet interrupter the CPlf). the redi rnitine weps testing ine flay until it is set hy the timer interrupt. This technique allows Abiplication rnirams to synchronize themiselves to the external clock and produce a constant sampling rate for a

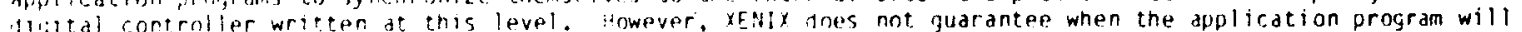

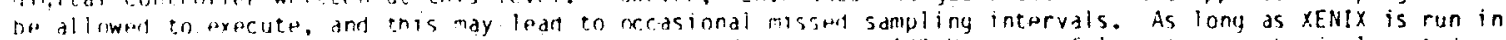

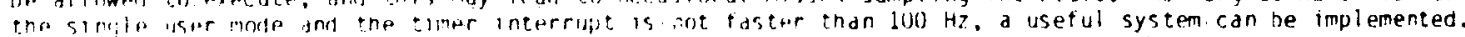

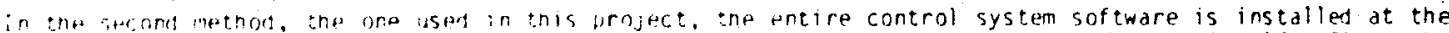

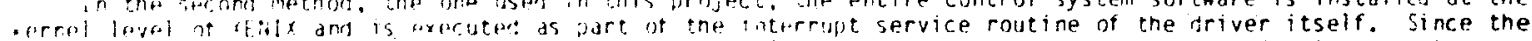

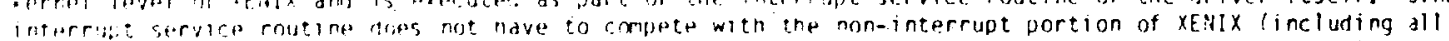

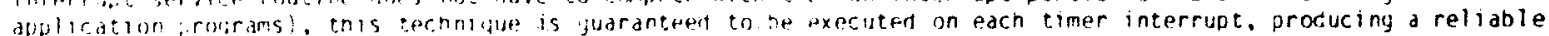

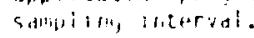

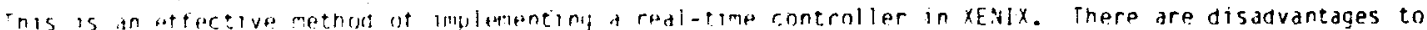

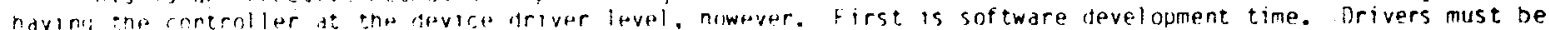

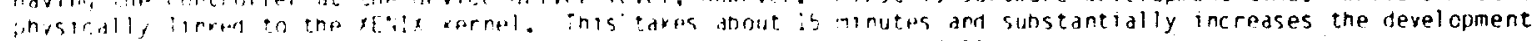

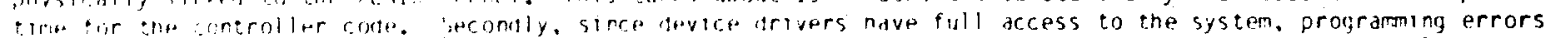

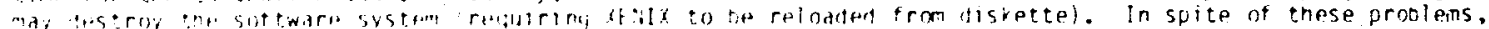

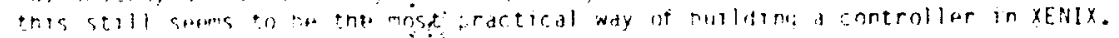

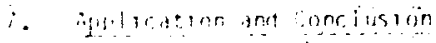

$\therefore \quad \therefore+11+\cdots+\cdots$

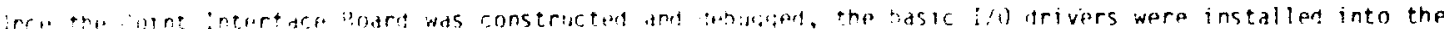

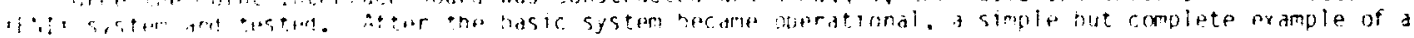


The ohjective of this projec: was to destgn and construct the hardware and interface sof tware to implement

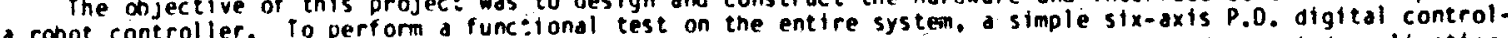
ler as implene.ted. In addition to testing the integrated system, it also served as docimented application guide for use of the JiB and the XENIX interface.

Figure a shows the bastc controller system. The controller is divided into five distinct subsystems: (1) the application sof tware which issues high level joint motion comands (kinematics, path planning. etc.) and

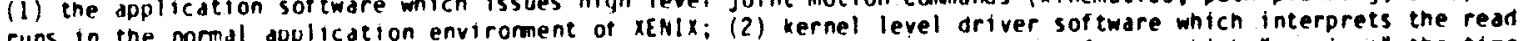
runs in the normal application enviroment of keNix; (2) kernel level driver sofeware which "services" the timer and write comands from the application programs: (3) Interrupt level driver sof tware which "services the timer interrupt by executing the control structure software, reading and writing directly to the jib hardware; (4) the joint inisface Bodrd wich interfaces the computer joint motor signals: and (5) the robot arm itself. theluding power anplifiers, joint motors and feedback elements.

The simple p. controller implemented in this project was able to satisfactorily control all six puma 560 joint motors simul taneously. The P.D. coefficients were experimentally determined by trial and error. This was joint motors simultanenusly. When dll joints were operated tonether. the stronil

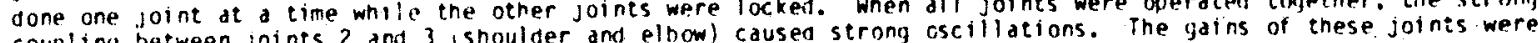

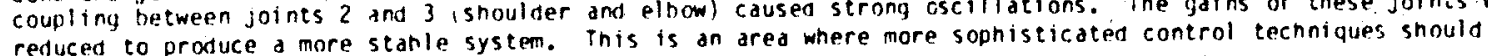
produce improved results.

\subsection{Conclision}

The basic objective of designing and constructing a general purpose robotic controller was completed successfully. The system has been used to control the puMA 560 rohot arms. demonstrating the functionality and flexibility of the design. The Jrint Interface Bodrd has served its overall design objective well.

Using the XeNix operatiny system was done with mixed results. High level sof tware is easily developed (at least for UNix users). Whereas the method of low-level servo-loop sof tware programing was somewhat less than desirable in that routines on this level must be directly linked (using the "ld" linked) to the xeNix kernel. desirable in that routines an this time-consuming task. XENIx also pronibits writing C-code in the kernel level Therefore. it involves a fairly time-consuming task. Xevix also pronibits writing C-code in ethe kernel las was disappointing, but which uses the flodting point coprocessor (via an undocunented c-compiler flag). This was diste and effort would be useful.

H. keforences

[1] 0. G. Binn. I Universal Six Joine Robot Controller, M.S. Thesis, Department of Electrical and Computer Enyineering, IIniversity of California. Davis. 1936.

[2] Intel Corporation. System 311! Installation an Operation Guide, 1).N. 173211-002. 0ctober 1983.

(3) Intel Corporation, XE!IX 286 Reference Manual. O.N. 174390-1008, 1944 .

[4] Intel coiporation. Intronuction to the iAPX 286, 0.N. 210308-1101. 1945.

[5] Intel Corworation, Introdurtion to the iApx 287, 1985.

[6] Intel Corpuration. Intel iultibus Specificatian. 0.i. 9yo0683. 19/8.

[7] Analog Devices inc.. "Fast, Complete 12-bit A/0 Converter with Microprocessor interface," Data-Acquisition

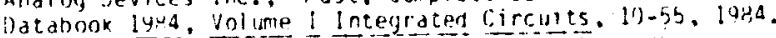

[3] Intel Corporation, "P254 programmanle interval Timer." mirerosystam fompnnents Handhook. Volume Il, $5-240$.

[y] Inimation, "5ijo Series Electrical orawiny Set tor val li ana lal olus Operating systems." linimate puma "ark 11 Robot. 394AC1. July 1985 .

[10] Intel Corporation, XENIX Device Driver Guide, 0.9. 1/4343-1301, I445. 

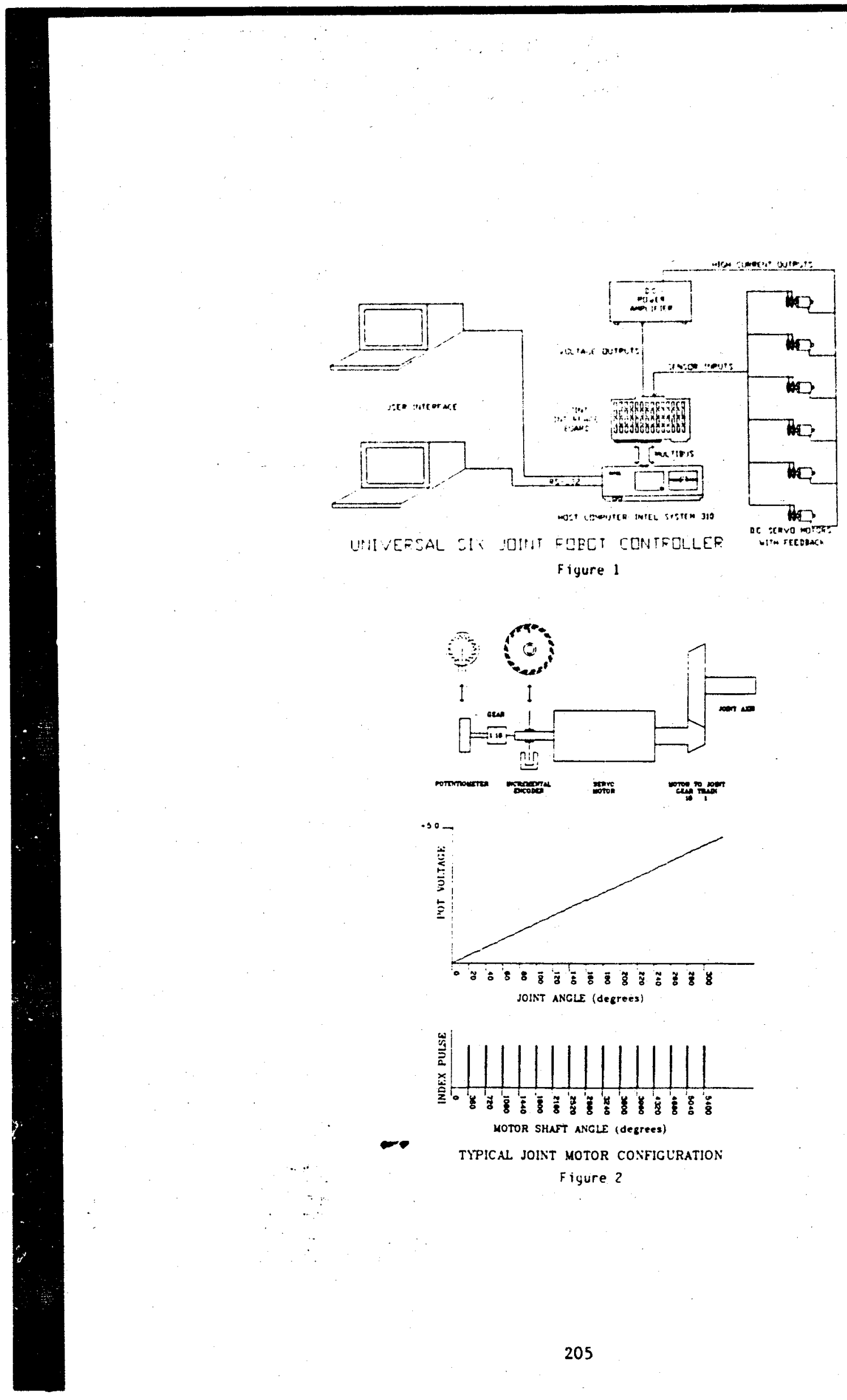

Figure 1
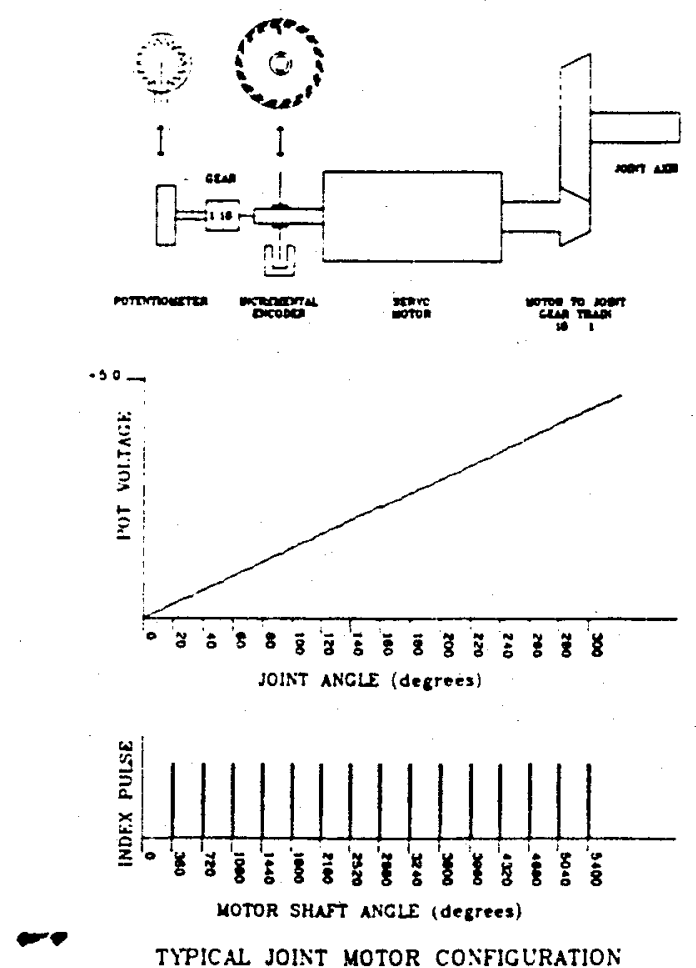

Figure? 


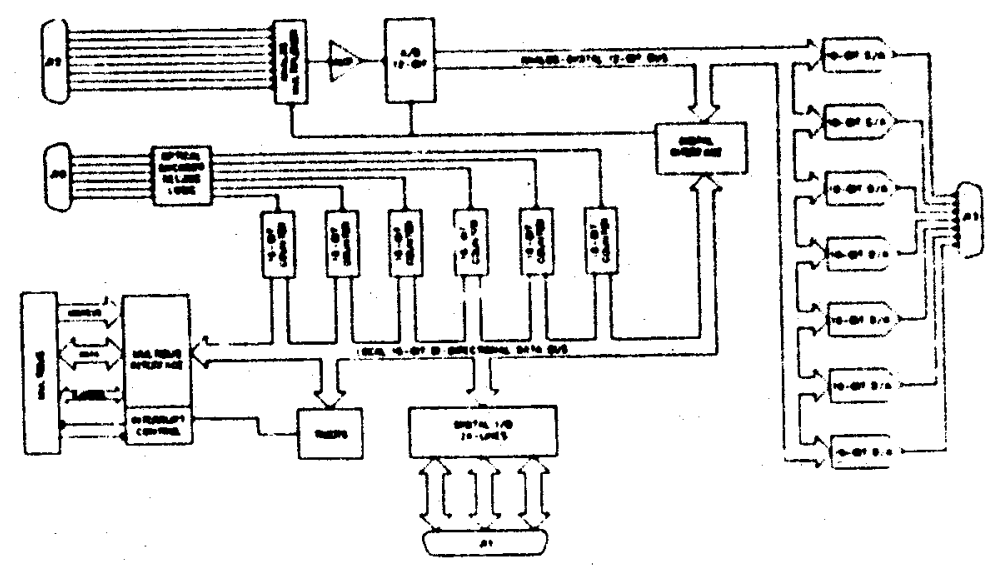

JOINT INTERFACE DOARD BLOCK DIAGRAM

flgure 3

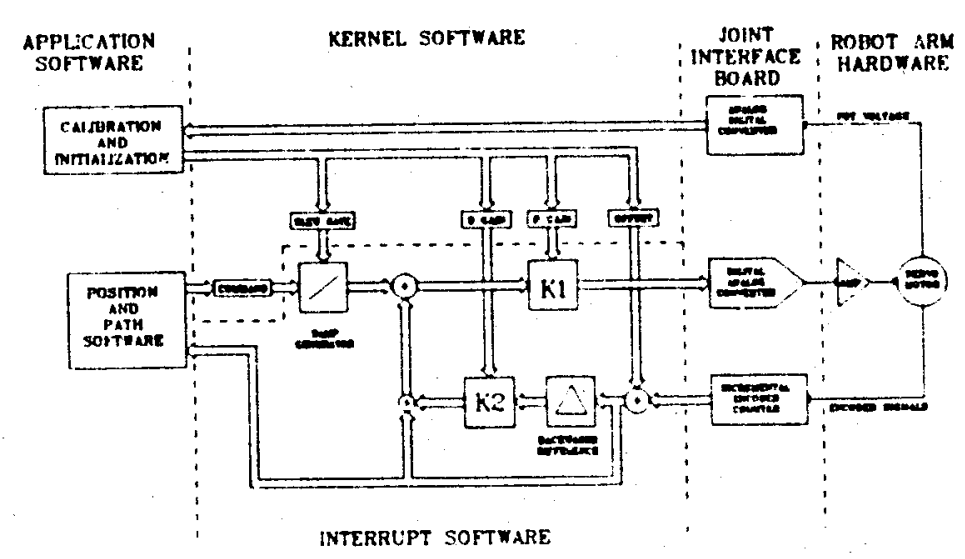

CONTKOIIER IOGICAL DIAGRAM

Figure 4 